\title{
Efficient Space-Time Block Codes Derived from Quasi-Orthogonal Structures
}

\author{
Mohammad Janani and Aria Nosratinia, Senior Member, IEEE
}

\begin{abstract}
We propose a new class of block codes that outperforms known space-time block codes at low rates. The new codes are designed by using appropriate rotations and set partitioning on two quasi-orthogonal codes, and combining subsets of their codewords. Using these techniques we are able to obtain higher coding gain at a given rate and improve performance. Simulations confirm the advantages of this code compared to other codes operating at the same rate and signal-to-noise ratio (SNR). We also provide an efficient maximum likelihood (ML) decoding algorithm for the new code.
\end{abstract}

Index Terms-Diversity, space-time coding, channel coding, wireless communications.

\section{INTRODUCTION}

W E design a new class of codes with excellent performance which are derived from a quasi-orthogonal structure (but are not quasi-orthogonal codes).

Quasi-orthogonal space-time codes (QOSTC) [1] were designed with an eye towards low-SNR performance while having simple decoding complexity. However, QOSTC could not achieve full diversity. A pairwise constellation rotation was proposed [2], [3] to overcome this problem. It is possible to apply the constellation rotation to either of the two parts of the quasi-orthogonal code, so two different families of quasiorthogonal codes can be obtained for the case of, e.g., four transmit antennas.

We design a new code by combining these modified quasiorthogonal codes and then pruning codewords from the combined code to achieve superior coding gain. To be specific, we choose our codewords from among a superset consisting of the union of two modified quasi-orthogonal codes. The pruning is achieved by a set partitioning algorithm applied to each of the constituent quasi-orthogonal codes. This set partitioning is inspired by the techniques first proposed by [4] and further enhanced by the authors in [5]. The former method is specifically for QOSTC and the second one is the general space-time code set partitioning method.

As a result of the manner in which we construct our code, the overall design is no longer quasi-orthogonal and therefore it does not directly inherit the easy decoding enjoyed by quasi-orthogonal codes. Therefore, we propose an efficient ML decoding algorithm whose average complexity is close to twice the complexity of the quasi-orthogonal decoder of

Manuscript received September 27, 2005; revised May 3, 2006; accepted June 10, 2006. The associate editor coordinating the review of this paper and approving it for publication was M. Uysal. This work was presented in part in ICC 2006.

The authors are with the Department of Electrical Engineering, University of Texas at Dallas, Richardson, TX 75083 (e-mail: \{janani, aria\}@utdallas.edu.

Digital Object Identifier 10.1109/TWC.2007.05764. similar rate. The performance of the new code, in terms of error rate, is superior to any of the block space-time codes we tested, when compared at the same rate and SNR. This includes enhanced quasi-orthogonal codes and the enhanced LD codes of [6].

\section{A. Notation}

We use the following notation throughout this paper. Uppercase bold letters denote matrices, for example codewords are denoted with $\mathbf{X}, \mathbf{Y}, \mathbf{Z}$ and unitary transforms are denoted with $\mathbf{U}$ which we concisely (but not entirely accurately) refer to as "rotations" in the sequel. Script letters denote sets of codewords, e.g. $\mathcal{S}, \mathcal{T}$. Subscripts are used to denote set partitioning. For convenience we define the multiplication of a set and a matrix, for example $\mathcal{S} \mathbf{U}$, as a new set whose members are the members of $\mathcal{S}$ each multiplied by $\mathbf{U}$.

The function $\mathrm{D}(\cdot, \cdot)$ computes the distance between two sets of codewords. With an abuse of notation we may see a codeword as one of the arguments of this function, which represents the set consisting of that single codeword.

\section{B. System Model}

The system model consists of a MIMO system with $L_{t}$ transmit and $L_{r}$ receive antennas. A flat fading channel is assumed, where the channel gains are constant during each fading interval and independent in successive intervals. The received signal, denoted by a $T \times L_{r}$ matrix $\mathbf{R}$, after matched filtering has the following form: $\mathbf{R}=\sqrt{\rho / L_{t}} \mathbf{X H}+\mathbf{N} . T$ represents the number of time slots for transmitting one block of symbols. $\rho$ is the average received signal-to-noise ratio per antenna. The matrix $\mathbf{S}$ is a QOSTB codeword of size $T \times L_{t}$. The channel matrix $\mathbf{H}=\left\{h_{i j}\right\}$ has the size of $L_{t} \times L_{r}$ where $h_{i j}$ is the fading channel coefficient between $j$ th received antenna and $i$ th transmit antenna. The AWGN is shown by the matrix $\mathbf{N}$. The receiver employs maximum likelihood (ML) decoding with perfect knowledge of channel state information.

\section{Modified Quasi-Orthogonal Space-Time Codes}

We focus on the case of $L_{t}=4$ where each codeword transmits four symbols at four timing slots. We start with the modified QOSTC [2], which achieves full diversity. The modified code applies a constellation rotation either on the first pair or the second pair of symbols. The code structure is

$$
X\left(\phi_{1}, \phi_{2}\right)=\left(\begin{array}{cccc}
e^{j \phi_{1}} x_{1} & e^{j \phi_{1}} x_{2} & e^{j \phi_{2}} x_{3} & e^{j \phi_{2}} x_{4} \\
-e^{-j \phi_{1}} x_{2}^{*} & e^{-j \phi_{1}} x_{1}^{*} & -e^{-j \phi_{2}} x_{4}^{*} & e^{-j \phi_{2}} x_{3}^{*} \\
e^{j \phi_{2}} x_{3} & e^{j \phi_{2}} x_{4} & e^{j \phi_{1}} x_{1} & e^{j \phi_{1}} x_{2} \\
-e^{-j \phi_{2}} x_{4}^{*} & e^{-j \phi_{2}} x_{3}^{*} & -e^{-j \phi_{1}} x_{2}^{*} & e^{-j \phi_{1}} x_{1}^{*}
\end{array}\right)
$$


where $x_{1}, x_{2}, x_{3}$, and $x_{4}$ are symbols from the constellation considered and $\phi_{1}$ and $\phi_{2}$ are the rotation angles. For each pair $\left(\phi_{1}, \phi_{2}\right)$, matrices $X\left(\phi_{1}, \phi_{2}\right)$ constitute a complete quasiorthogonal codebook. Thus by changing $\left(\phi_{1}, \phi_{2}\right)$ we can generate a family of codebooks, to be used in our design process below.

\section{Distance Criterion}

We use the Coding Gain Distance (CGD) as the design criterion, which we introduce below in a manner similar to [7]. For two codewords $\mathbf{X}$ and $\mathbf{Y}$ construct $\mathbf{A} \triangleq(\mathbf{X}-\mathbf{Y})(\mathbf{X}-$ $\mathbf{Y})^{H}$, and then define CGD $=\operatorname{det}(\mathbf{A})$. By extension, the minimum CGD of a codebook $\mathcal{S}$ is defined as the minimum of CGD of all non-identical codeword pairs in $\mathcal{S} \times \mathcal{S}$. Similarly the distance between two codebooks $\mathcal{S}$ and $\mathcal{T}$ is $\mathrm{D}(\mathcal{S}, \mathcal{T})=$ $\min \operatorname{det}(\mathbf{A}(\mathbf{X}, \mathbf{Y}))$.

\section{Code Design}

To design the new codebook, we start from a union of quasi-orthogonal codebooks, which obviously has more code vectors than we need for our desired rate. Then we use pruning to reduce the number of codewords in a way that the minimum distance of the codebook is increased, and thus the performance of the code is improved.

In particular, we start with the union of two quasiorthogonal codebooks $\mathcal{T} \cup \mathcal{S}$, where $\mathcal{S}=\left\{X\left(\phi_{1}, 0\right)\right\}$ and $\mathcal{T}=\left\{X\left(0, \phi_{2}\right)\right\}$. Since $\mathcal{S} \cup \mathcal{T}$ has twice as many codewords, the union is pruned down by one-half to arrive at the new codebook $\mathcal{C}$, which has the original rate.

Clearly the performance of the new codebook $\mathcal{C}$ is bounded below by the performance of quasi-orthogonal codes $\mathcal{S}$ and $\mathcal{T}$, because each of them is one possible pruning of $\mathcal{S} \cup$ $\mathcal{T}$. Therefore, this process can only improve the code. The empirical fact is that it indeed does so in a significant way, and the resulting code is better, for example in the case of BPSK it is better by $1.3 \mathrm{~dB}$.

Next, we explore the pruning of the superset $\mathcal{S} \cup \mathcal{T}$. The most general (globally optimal) pruning of this superset would be very difficult. Instead, we present one systematic way of doing so. We note that the new codebook can be written as $\mathcal{C}=\mathcal{S}^{*} \cup \mathcal{T}^{*}$, where $\mathcal{S}^{*}$ and $\mathcal{T}^{*}$ are the surviving members of $\mathcal{S}$ and $\mathcal{T}$ after pruning. It is evident that if the code $\mathcal{C}$ is to be a good code, then constituent codes $\mathcal{S}^{*}$ and $\mathcal{T}^{*}$ must also have good distance properties.

The above observation suggests a technique for designing the new code. We first partition the codebooks $\mathcal{S}$ and $\mathcal{T}$ such that each partition has good distance properties. Then we combine partitions in a judicious way to construct $\mathcal{C}$.

In order to clarify the method, consider a code design example for BPSK symbols where $\phi_{1}=\phi_{2}=\pi / 2$ is the optimum value. The set partitioning of the code $\mathcal{S}$ into two subcodes gives

$$
\begin{gathered}
\mathcal{S}_{1}=\{0000,0011,0101,0110,1001,1010,1100,1111\} \\
\mathcal{S}_{2}=\{0001,0010,0100,0111,1000,1011,1101,1110\}
\end{gathered}
$$

where each codeword has been represented by its four binary symbols $\left(x_{1}, x_{2}, x_{3}, x_{4}\right)$. In the same manner, the code $\mathcal{T}$ can be set partitioned into $\mathcal{T}_{1}$ and $\mathcal{T}_{2}$, which are, respectively, similar to $\mathcal{S}_{1}$ and $\mathcal{S}_{2}$ with the second pairs in each codeword rotated by $\pi / 2$.

Table I shows the inter subcode distances. The minimum CGD of the new code $\mathcal{S}$ or $\mathcal{T}$ is

$$
D\left(\mathcal{S}_{1}, \mathcal{S}_{2}\right)=D\left(\mathcal{T}_{1}, \mathcal{T}_{2}\right)=256 .
$$

Looking at the inter-distances of subcodes, we can pick $\mathcal{S}_{1}$ from $\mathcal{S}$ and $\mathcal{T}_{2}$ from $\mathcal{T}$ to form a new code. The minimum CGD of the code has risen up to

$$
D\left(\mathcal{S}_{1}, \mathcal{T}_{2}\right)=2304
$$

which is much greater than the distance of the code $\mathcal{S}$ or $\mathcal{T}$. This guarantees better performance.

\section{A. Augmented Code Design}

Unfortunately we found that for higher rate codes, the pick-and-choose method alone does not always improve the distance properties of the original (constituent) codebooks. In other words, the quasi-orthogonal families mentioned above do not always provide a sufficiently rich set of codewords to choose from. In order to overcome this limitation, we allow the rotation of the constituent codebooks. To preserve quasiorthogonality of the constituent codebooks, we use unitary operations, that is:

$$
\mathcal{T} \leftarrow \mathcal{T} \mathbf{U},
$$

where $\mathrm{U}$ is a unitary matrix.

More specifically, we propose to use a diagonal unitary matrix, for the following reason. One of the properties of the QOSTC is that the transmitted power from each antenna at each transmission time is one. Using a diagonal unitary matrix preserves this property. In other words, peak-to-average transmitted power at each time instance remains one. Thus,

$$
\mathbf{U}=\operatorname{diag}\left(e^{j \theta_{1}}, e^{j \theta_{2}}, e^{j \theta_{3}}, e^{j \theta_{4}}\right),
$$

where $\operatorname{diag}(\cdot)$ denotes a diagonal matrix.

A unitary rotation does not change the distance property of a code. Therefore, set partitioning of the code still remains the same, but each subcode is rotated. Now we can build the new code. We can pick one subcode from $\mathcal{S}$ and one rotated subcode from $\mathcal{T}$. The goal is to design a good unitary matrix that increases the minimum distance of the code, in other words

$$
\max _{\mathbf{U}} D\left(\mathcal{S}_{1}, \mathcal{T}_{2} \mathbf{U}\right)
$$

U could be obtained by exhaustive search. Table II shows the cross distances for QPSK constellation and $\mathbf{U}=\operatorname{diag}\left(e^{j 0.9 \pi}, e^{j 1.1 \pi}, e^{j 1.6 \pi}, e^{j 0.4 \pi}\right)$. As shown, $\mathbf{U}$ helps to increase the minimum distance from 16 to 43.94 .

\section{Decoding Algorithm}

One of the advantages of quasi-orthogonal codes is simple decoding. In particular, each quasi-orthogonal codeword consists of two types of sub-matrices, each of them possibly an orthogonal or quasi-orthogonal codeword of lower dimension. The standard decoding process for quasi-orthogonal codes allows the distance metric to be written as a sum of metrics 
TABLE I

CGD OF THE SET PARTITIONS FOR BPSK CONSTELLATION

\begin{tabular}{|c|cccc|}
\hline$D(\cdot, \cdot)$ & $\mathcal{S}_{1}$ & $\mathcal{S}_{2}$ & $\mathcal{T}_{1}$ & $\mathcal{T}_{2}$ \\
\hline $\mathcal{S}_{1}$ & 0 & 256 & 0 & 2304 \\
$\mathcal{S}_{2}$ & 256 & 0 & 2304 & 0 \\
$\mathcal{T}_{1}$ & 0 & 2304 & 0 & 256 \\
$\mathcal{T}_{2}$ & 2304 & 0 & 256 & 0 \\
\hline
\end{tabular}

1) Decode with quasi-orthogonal codebook $\mathcal{S}$, arriving at $\hat{\mathbf{X}}_{s}$.

2) Decode with quasi-orthogonal codebook $\mathcal{T}$, arriving at $\hat{\mathbf{X}}_{t}$.

3) If $\hat{\mathbf{X}}_{s}$ or $\hat{\mathbf{X}}_{t}$ (but not both) belongs to $\mathcal{C}$, it is declared the decoded codeword.

4) If both belong to $\mathcal{C}$, then the one with the better ML metric is declared the decoded codeword.

5) If neither $\hat{\mathbf{X}}_{s}$ nor $\hat{\mathbf{X}}_{t}$ belongs to the new codebook $\mathcal{C}$, do exhaustive search.

Fig. 1. Decoding algorithm

for these two sections, thus simplifying the ML detection. We refer to this construction of the metric, and the corresponding detection algorithm, as quasi-orthogonal decoding [7].

For our new code, we are interested to maintain, as much as possible, the simple decoding afforded by the quasi-orthogonal structure, while achieving the performance of ML decoding. In general the new codebook $\mathcal{C}$ does not by itself present a direct way of simple ML decoding. However, our knowledge that the codewords of $\mathcal{C}$ come from either $\mathcal{S}$ or $\mathcal{T}$, can be used to construct an efficient decoding algorithm.

The decoding algorithm is motivated by the conditioning ideas that have been used, e.g., in sphere decoding. Consider that each received codeword either belongs to $\mathcal{S}$ or to $\mathcal{T}$. Of course the decoder does not know a-priori which is the case. Therefore, the decoder performs two quasi-orthogonal detections, according to codebooks $\mathcal{S}$ and $\mathcal{T}$, resulting in two codeword candidates $\hat{\mathbf{X}}_{s}$ and $\hat{\mathbf{X}}_{t}$ respectively. We then check to see if either or both of these two candidates are actually members of $\mathcal{C}$. If exactly one is a valid codeword, the decoding is successful. If both are valid codewords, then we choose the one that has the better ML metric. If neither is a valid codeword, we have no choice but to perform a wider search on $\mathcal{C}$. However, thankfully the probability of this last event is very small for reasonable codeword error rates, and thus the average complexity of decoding is minimally impacted by the last case. The flow of decoding is summarized in Figure 1.

The computational complexity of this decoder depends on how often quasi-orthogonal decoding produces a valid codeword. Whenever that happens, no further action is required and thus a codeword has been cheaply detected. Whenever it fails, the decoder can either declare failure (similar to the concept of a bounded distance decoder) or if we wish to build a complete decoder, we must search more extensively among codeword candidates. Thankfully the probability of this event is low unless the codeword error probability is high. For example, simulations show that for a $4 \times 1$ system employing a BPSK constellation, at codeword error rates $P_{e}=10^{-2}$ and
TABLE II

CGD OF THE SET PARTITIONS FOR QPSK CONSTELLATION

\begin{tabular}{|c|cccc|}
\hline$D(\cdot, \cdot)$ & $\mathcal{S}_{1}$ & $\mathcal{S}_{2}$ & $\mathcal{T}_{1}$ & $\mathcal{T}_{2}$ \\
\hline $\mathcal{T}_{1}$ & 0 & 2.33 & 0 & 16 \\
$\mathcal{T}_{2}$ & 2.33 & 0 & 16 & 0 \\
$\mathcal{T}_{1} \mathbf{U}$ & 0 & 43.94 & 0 & 16 \\
$\mathcal{T}_{2} \mathbf{U}$ & 43.94 & 0 & 16 & 0 \\
\hline
\end{tabular}

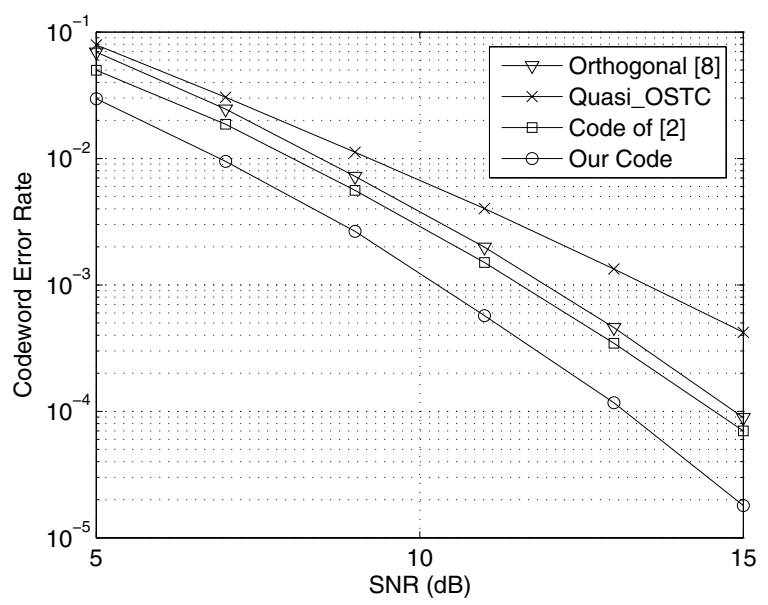

Fig. 2. $L_{t}=4, L_{r}=1$, and BPSK symbols.

$P_{e}=10^{-3}$ the probability of being forced into exhaustive search are only $P_{e x}=0.0185$ and $P_{e x}=0.0021$ respectively.

We now proceed to calculate the metric for the pairwise decoding. Each codeword of the new code belongs to one of the quasi orthogonal codebooks. The decoding metric after simplification is

$$
f_{13}\left(x_{1}, x_{3}, \phi_{1}, \phi_{2}, \mathbf{U}\right)+f_{24}\left(x_{2}, x_{4}, \phi_{1}, \phi_{2}, \mathbf{U}\right),
$$

where

$$
\begin{aligned}
& f_{13}\left(x_{1}, x_{3}, \phi_{1}, \phi_{2}\right)=\left(\sum_{i=1}^{4}\left|h_{i}\right|^{2}\right)\left(\left|x_{1} e^{j \phi_{1}}\right|^{2}+\left|x_{3} e^{j \phi_{2}}\right|^{2}\right) \\
& -2 \Re\left\{x_{1} e^{j \phi_{1}}\left(r_{1}^{*} h_{1} e^{j \theta_{1}}+r_{2} h_{2}^{*} e^{-j \theta_{2}}+r_{3}^{*} h_{3} e^{j \theta_{3}}+r_{4} h_{4}^{*} e^{-j \theta_{4}}\right)\right. \\
& \left.+x_{3} e^{j \phi_{2}}\left(r_{1}^{*} h_{3} e^{j \theta_{3}}+r_{2} h_{4}^{*} e^{-j \theta_{4}}+r_{3}^{*} h_{1} e^{j \theta_{1}}+r_{4} h_{2}^{*} e^{-j \theta_{2}}\right)\right\} \\
& +4 \Re\left\{x_{1} x_{3}^{*} e^{j\left(\phi_{1}-\phi_{2}\right)}\right\} \Re\left\{h_{1} e^{j \theta_{1}} h_{3}^{*} e^{-j \theta_{3}}+h_{2} e^{j \theta_{2}} h_{4}^{*} e^{-j \theta_{4}}\right\}
\end{aligned}
$$

and

$$
\begin{aligned}
& f_{24}\left(x_{2}, x_{4}, \phi_{1}, \phi_{2}\right)=\left(\sum_{i=1}^{4}\left|h_{i}\right|^{2}\right)\left(\left|x_{2} e^{j \phi_{1}}\right|^{2}+\left|x_{2} e^{j \phi_{2}}\right|^{2}\right) \\
& -2 \Re\left\{x_{2} e^{j \phi_{1}}\left(r_{1}^{*} h_{2} e^{j \theta_{2}}-r_{2} h_{1}^{*} e^{-j \theta_{1}}+r_{3}^{*} h_{4} e^{j \theta_{4}}-r_{4} h_{3}^{*} e^{-j \theta_{3}}\right)\right. \\
& \left.+x_{4} e^{j \phi_{2}}\left(r_{1}^{*} h_{4} e^{j \theta_{4}}-r_{2} h_{3}^{*} e^{-j \theta_{3}}+r_{3}^{*} h_{2} e^{j \theta_{2}}-r_{4} h_{1}^{*} e^{-j \theta_{1}}\right)\right\} \\
& +4 \Re\left\{x_{2} x_{4}^{*} e^{j\left(\phi_{1}-\phi_{2}\right)}\right\} \Re\left\{h_{1} e^{j \theta_{1}} h_{3}^{*} e^{-j \theta_{3}}+h_{2} e^{j \theta_{2}} h_{4}^{*} e^{-j \theta_{4}}\right\},
\end{aligned}
$$

where $\Re\{\cdot\}$ indicates the real part of a complex number. We note that for many practical cases, such as BPSK modulation where $\theta_{i}=0$, many of the terms above disappear and the calculations simplify significantly.

\section{Simulations}

We assume a slow fading channel and one receive antenna. Figure 2 shows the codeword error rate for the rate-one code, 


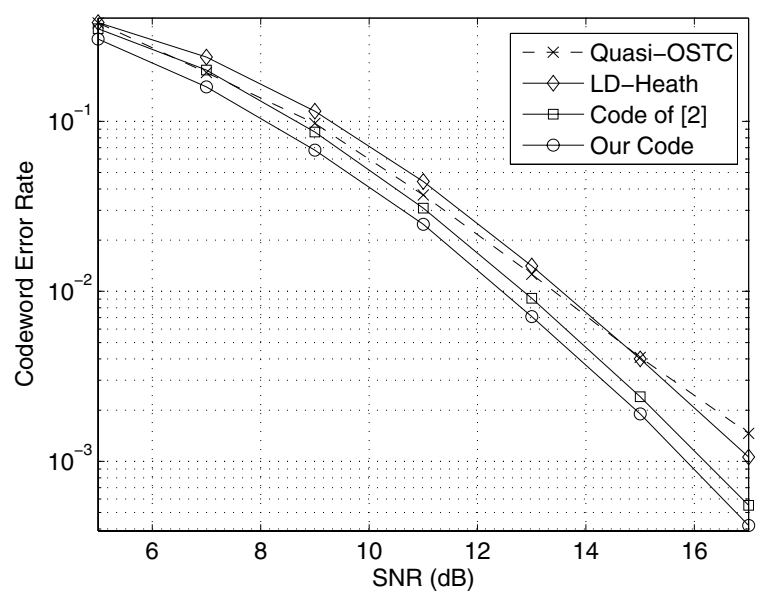

Fig. 3. $L_{t}=4, L_{r}=1$, and QPSK symbols.

which uses BPSK modulation. At codeword error rate of $10^{-4}$, gains of $1.3 \mathrm{~dB}$ and $1.7 \mathrm{~dB}$ have been obtained over modified quasi-orthogonal space time code [2] and orthogonal space time code [8], respectively.

Figure 3 depicts the performance of the code for QPSK. The parameters of the new codes are $\phi_{1}=\phi_{2}=\pi / 4$ with $\mathbf{U}=\operatorname{diag}\left(e^{j 0.9 \pi}, e^{j 1.1 \pi}, e^{j 1.6 \pi}, e^{j 0.4 \pi}\right)$. The code has 0.5 and $1.1 \mathrm{~dB}$ gain respectively over modified QOSTC [2] and LD codes of [6].

\section{Conclusion}

We propose a new space-time code whose codewords are derived from two different quasi-orthogonal space-time codes (QOSTC). Employing constellation rotations on different pairs of QOSTC gives these two sets. We select the codewords from these two sets through set partitioning in order to increase the minimum codeword pairwise distance. The resulting codes exhibit very good performance.

\section{REFERENCES}

[1] H. Jafarkhani, "A quasi-orthogonal space-time code," IEEE Trans. Commun., vol. 49, no. 1, pp. 1-4, Jan. 2001.

[2] N. Sharma and C. B. Papadias, "Improved quasi-orthogonal codes through constellation rotation," IEEE Trans. Commun., vol. 51, no. 3, pp. 563-571, Mar. 2003.

[3] W. Su and X. Xia, "Signal constellations for quasi-orthogonal spacetime block codes with full diversity," IEEE Trans. Inf. Theory, vol. 50, no. 10, pp. 2331-2347, Oct. 2003.

[4] H. Jafarkhani and N. Hassanpour, "Super-quasi-orthogonal space-time trellis codes for four transmit antennas," IEEE Trans. Wireless Commun., vol. 4, no. 1, pp. 215-227, Jan. 2005.

[5] M. Janani and A. Nosratinia, "Generalized block space-time trellis codes: set parttioning and code design," in Proc. IEEE WCNC 2005, pp. 461465.

[6] R. W. Heath and A. J. Paulraj, "Linear dispersion codes for MIMO systems based on frame theory," IEEE Trans. Signal Processing, vol. 50, no. 10, pp. 2429-2441, Oct. 2002.

[7] H. Jafarkhani and N. Seshadri, "Super-orthogonal space-time trellis codes," IEEE Trans. Inf. Theory, vol. 49, no. 4, pp. 937-950, Apr. 2003.

[8] V. Tarokh, H. Jafarkhani, and A. R. Calderbank, "Space-time block codes from orthogonal designs," IEEE Trans. Inf. Theory, vol. 45, no. 5, pp. 1456-1467, July 1999. 